\title{
Dysfunction of the lower gastrointestinal tract as a complication of diabetes - a problematic issue in the diabetic care
}

\section{ABSTRACT}

The purpose of this article is to familiarize the reader with abnormalities concerning the lower gastrointestinal tract in patients with diabetes and to present the latest views related to their pathogenesis and possibilities of treatment as well as subjects of future research. Gastrointestinal disorders as a complication of diabetes are common. Disorders of the upper gastrointestinal tract are diagnosed most often; lower gastrointestinal tract symptoms are equally frequent, but, due to their embarrassing nature, patients are less likely to report them. They are based on complex mechanisms, not only autonomic neuropathy, as was believed for years. Treatment is complex, much like pathogenesis. Currently there is no effective treatment and it is mostly empirical. Discovering new mechanisms of the disease opens the opportunity for further research whose aim is to develop new therapeutic options. (Clin Diabet 2016; 5, 3: 100-106)

Key words: diabetes mellitus, diabetic neuropathy, diabetic complications, constipation, diarrhea, oxidative stress

\section{Introduction}

In recent years, knowledge concerning mechanisms underlying gastroenterological disorders in diabetes has

Address for correspondence: Lek. Małgorzata Reszczyńska Uniwersytecki Szpital Kliniczny ul. Borowska 213, 50-556 Wrocław e-mail: g.reszczynska@gmail.com Translation: GROY Translations Clinical Diabetology 2016, 5, 3, 100-106 DOI: $10.5603 /$ DK.2016.0017

Received: 04.12.2015 considerably expanded. It is thought that beyond commonly diagnosed autonomic neuropathy (AN), other mechanisms influence their occurrence [1]. Diabetic neuropathy significantly decreases patients' quality of life. It is a recognized factor in development of diabetic foot, as well as death [2]. Patients with diabetes often (up to $75 \%$ ) report gastrointestinal symptoms $[3,4]$. The nature of these disorders is atypical, which slows down the diagnosis and significantly impedes its treatment [5]. It is known that AN occurs often and the knowledge concerning mechanisms underlying it is expanding; however its etiology is highly complex $[6,7]$. Subclinical diabetic autonomic neuropathy can occur within a year of diagnosis of type 2 diabetes and two years of diagnosis of type 1 diabetes, while first symptoms appear only after many years $[7,8]$. Taking recent findings into consideration, it is known that not only autonomic neuropathy alone is responsible for these complications; complex mechanisms underlying damage to the enteric nervous system, damage to the interstitial cells of Cajal, or damage to the smooth muscles are increasingly being cited as significant in etiopathogenesis of diabetic gastroenteropathy [1, 4].

\section{Epidemiology}

According to various researchers, symptoms from the upper gastrointestinal tract resulting from esophageal or gastric (gastroparesis) motility disorders are the most common manifestation of diabetic gastroenteropathy occurring in $50 \%$ and $40 \%$ of patients, respectively [7]. Symptoms stemming from complications concerning small and large intestine are diarrhea (20\%) [7-9] or constipation (according to various authors between $25 \%$ [7] and $60 \%$ [9] of patients), as well as other anorectal function disorders, e.g. fecal incontinence or visceral sensitivity disorders [10-12]. A lot of research concerning disorders of the upper gastrointestinal 
tract in patients with diabetes can be found in available literature; much less research, however, has been done concerning the lower gastrointestinal tract [11]. Taking into account considerable complexity and variety of mechanisms underlying gastrointestinal disorders in diabetes, there seems to be validity in the view of some researchers that the ineffectiveness of current treatment is due to the complexity of the problem and the fact that it is usually focused only on one of the many mechanisms [13].

\section{Physiology}

Complex regulatory mechanisms of gastrointestinal motility herald complex pathogenesis of its disorders in diabetes. Three basic structures play the main role in coordinating all processes: smooth muscles, which are directly responsible for motility, autonomic system and enteric nervous system (ENS) as well as interstitial cells of Cajal (ICC) - specialized muscle cells of the gastrointestinal tract which serve as a pacemaker and enable coordinated contraction of gastrointestinal muscles through synchronization of functions of nerves and muscles. ICC are present in the entire gastrointestinal tract, starting with esophagus and continuing to the internal anal sphincter. The final regulatory mechanism concerns the nervous system, i.e. ENS and, indirectly, the autonomic nervous system. This division of the nervous system is connected to ENS via efferent fibers, parasympathetic innervation from the nucleus of the vagus nerve and the sacral spinal cord segment and sympathetic innervation from the postganglionic nerves from the prevertebral ganglia [13]. ENS is an autonomous unit controlling and coordinating motility, blood flow and secretion in the gastrointestinal tract. Its elements are present in bile ducts, pancreas and all segments of the gastrointestinal tract. The system is composed of $10^{8}$ nerve cells forming two main plexuses - the Auerbach's plexus and the submucosal plexus as well as other nerve cells, e.g. enteric glial cells. These neurons are responsible for controlling gastrointestinal motility and secretion, in response to changes in smooth muscle tension and content of the gastrointestinal lumen. ENS is connected to the brain via afferent and efferent fibers [13, 14]. Control of the nerve cells depends on the release of neurotransmitters, both excitatory and inhibitory (e.g. NO, VIP — vasoactive intestinal polypeptide, ATP) [13].

In addition, gastrointestinal tract is closely linked to the autonomic nervous system; nearly all segments are innervated by sympathetic and parasympathetic efferent fibers and send signals to the parasympathetic system through afferent fibers. Due to the fact that autonomic neuropathy in diabetes in respect to other systems is well-known, gastrointestinal disorders have also been linked primarily to it. However, available results of research concerning the relation between symptoms of $A N$ in respect to other systems and symptoms concerning the gastrointestinal tract are controversial [1].

\section{Pathogenesis}

Pathogenesis of gastrointestinal disorders in diabetes stems from a dysfunction of one of the closely linked structures necessary for proper motility, or to a certain degree from of all of them [13].

It is thought that the following, among others, factors influencing pathogenesis of gastrointestinal disorders are: gastrointestinal motility disorders (due to disorders in gastrointestinal innervation), genetic factors, visceral hypersensitivity, hormonal disorders, dysregulation of the autonomic nervous system, hyperglycemia, inflammatory processes, nerve growth factors as well as autoimmune factor is also considered, particularly in etiology of gastroparesis [7-9, 14]. Disorders in microcirculation supplying nerve fibers should also be considered an important factor [7, 8, 15].

Mechanisms underlying neuropathy (autonomic and peripheral) in diabetes are highly complex, with a multifactorial etiology $[6,7,9,15]$. Autonomic neuropathy in diabetes is still a subject of intensive research, e.g. a research conducted by Vezeou et al. showed no relation between AN with occurrence of gastrointestinal symptoms, as well as no relation between fluctuations of glycemia with motility disorders in patients with type 1 diabetes; the research was, however, limited in certain ways [16]. In contrast, results of other research $[14,19,20]$ show a clear relationship. Increased risk of neuropathy in cases of type 1 diabetes related to longer duration of the disease, periods of high glucose concentration in the blood and gastrointestinal motility disorders are still the subjects for discussion [6].

Tests performed on rats show that during the course of diabetes an initial decrease in the number of neurons occurs, followed by their probable regeneration [14]. Research suggests that a reconstruction of inhibitory and excitatory neurons occurs in patients with diabetes, leading to a weakened muscular response in which nerve fibers are an intermediary, and which directly translates to observed gastrointestinal motility disorders. To a greater degree the damage most probably concerns inhibitory neurons, particularly nNOS (neuronal nitric oxide synthase), the number of which decreases in diabetes, as well as VIP and neuropeptide Y [18]. Reduced expression, or activity, of nNOS is probably the most consistent change in respect to ENS, although there is a research indicating an increase 
or no change in nNOS in patients with diabetes [13]. The nNOS neurons produce nitric oxide (NO), which is a non-adrenergic, non-cholinergic, primary, inhibitory neurotransmitter, intermediating in relaxation of gastrointestinal smooth muscles and therefore playing an important role in motility $[8,13]$. There is most likely an inhibition of nNOS expression by advanced glycation end products (AGEs) binding to nerve plexuses [9].

A number of research was conducted in order to find an answer to the question of what underlies these disorders. Currently it is known that high glucose concentration is the main cause of diabetes complications [7]. The second most important aspect is an intracellular overproduction of reactive oxygen and nitrogen species (RONS) [20]. These molecules induce damage to the cellular DNA and stimulate various repair processes. As a consequence, dysregulation and activation of various signals initiating cell death occur, such as advanced glycation end products (AGEs) [7]. High glucose concentration in the cell may also induce additional protein glycation [14]. AGEs are formed as a result of non-enzymatic reactions between aldehyde groups of reduced sugars and proteins, lipids and nucleic acids [15]. AGEs affect proteins by changing their structure and function; these processes take place both intra- and extracellularly, and they may, initiate a cascade of proinflammatory agents (e.g. IL-1, IL-6, TNF, etc.) through special receptors (RAGEs) and increase oxidative stress [7]. In research conducted by Takuma et al. it was shown that blocking RAGE receptors in mice resulted in reduced frequency of nerve complications [21]. Oxidative stress, which is greatly increased in diabetes, is currently considered an important factor in pathogenesis of gastrointestinal complications [9, 22]. Disorders in hexosamine and protein kinase C (PKC) transformation pathways also play an important role in RONS production [7]. An additional mechanism induced by hyperglycemia concerns formation of polyols - e.g. sorbitol, increased, non-physiological, concentrations of which lead to excessive consumption of NADH, which are necessary to regenerate antioxidants $[7,8]$. An imbalance in the NAD:NADH ratio can be a direct cause of neuron damage and/or reduced blood supply to the nerves [8].

Recent research highlights the role of inflammatory process in pathogenesis of diabetic autonomic neuropathy [7, 9]. Many factors (e.g. overproduction of proinflammatory cytokines) influence secondary damage to neurons and vessels; these processes remain related to oxidative stress. According to some research, inflammatory markers, e.g. CRP, IL-6, IL-8, TNF-alpha or endothelin-1, are considered predictive factors in development of cardiovascular autonomic neuropathy [7].
Nerve growth factors - neurotrophins also participate in pathogenesis of diabetic autonomic neuropathy [7-9]. They are also seen as new therapeutic targets [13]. One of them is IGF-1, level of which in both types of diabetes is reduced. This promotes smooth muscle atrophy and directly weakens gastrointestinal motility [23].

Among metabolic disorders, apart from the obvious role of hyperglycemia in development of neuropathy, disorders of lipid metabolism are also highlighted $[1,7,8,15]$. These disorders are a subject of a number of research conducted in recent years, e.g. Fakhir et al. showed evident relation between dyslipidemia and the development of neuropathy in patients with type 2 diabetes [23]. Lipid metabolism disorders as well as increased lipid peroxidation resulting from oxidative stress occurring in patients with diabetes, lead in the effect to the formation of advanced lipoxidation endproducts (ALEs), which show a chemical similarity to AGEs and have a similar effect [15]. However, free fatty acids, hypertriglyceridemia and reduced HDL cholesterol concentration may intermediate in oxidative and inflammatory processes damaging tissues and, according to some researchers, correlate with development of neuropathy [7]. Researchers have shown, on the basis of animal models, that a diet rich in lipids may lead to loss of enteric neurons [1].

Genetic predisposition towards oxidative stress and an increased risk of neuropathy developing in relation to polymorphism of antioxidant enzymes has been recently researched [13] and should also be mentioned here.

Among other causes, damage to microcirculation, both direct and indirect, resulting from aforementioned metabolic processes is highlighted $[7,8,15]$. Endotheliopathy (i.e. damage to the vessels' endothelium) is a fundamental cause of damage to the small vessels supplying peripheral nerves [15].

Based on performed post-mortem examinations and tests on animals, it is known that during the course of diabetes, disorders concerning smooth muscles occur. It is still unclear why atrophic changes can be observed in the upper gastrointestinal tract and the hypertrophic changes in the lower tract [13].

The role of ICC has been discovered fairly recently [13]. Apart from producing independent impulses partially responsible for gastrointestinal motility, it appears that these cells act as intermediaries of nerve signals between motor neurons, efferent fibers from the autonomic nervous system, and muscle fibers [1]. A view that both inhibitory and excitatory neurotransmission between ENS and smooth muscle cells is intermediated by a special type of interstitial cells of Cajal - ICC-IM 
- has been proposed [1]. Based on several tests conducted on patients with diabetes and animal models, a decrease in the number of these cells was observed [13]. For example, in a research conducted by He et al. a clear lack of ICC was proven during a biopsy of small intestine in a patient with a prolonged, poorly controlled type 1 diabetes complicated by neuropathy [24]. Summarizing results of available research, it is known that a decrease in the number of ICC is a very common disorder in patients with gastrointestinal complications in both type 1 and type 2 diabetes. It is most likely not an isolated phenomenon and is accompanied by dystrophy of nerves and smooth muscles [13]. The degree of these changes appears to correlate with duration and severity of diabetes and symptoms of gastroenteropathy [13]. Mechanisms underlying damage to these cells require further research. Of interest is a report by researchers who showed that hyperglycemia per se is not enough to damage ICC and, generally speaking, deficiency of growth factors, e.g. IGF-1, and of insulin itself, is probably more significant [1].

Among others, new pathogenetic factors which are a subject of intensive research, intestinal microflora, microRNA as well as autophagy theory are worth mentioning [1]. It has been shown that experimentally removed large intestine microflora causes cecal distention due to bad motility and recreating the microflora causes this effect to disappear [25]. It is known that slowed down gastrointestinal motility in diabetes can lead to abnormalities in the intestinal microflora, which in turn can affect neurotransmissions between the gastrointestinal tract and the central nervous system and these observations are a subject of ongoing research [1].

\section{Symptoms}

The symptoms of diabetes complications in respect to lower gastrointestinal tract are taking the form of diarrhea, constipation or anorectal disorders occur fairly often in patients with diabetes $[26,27]$; according to various sources from $60 \%$ [3] and up to $76 \%$ [28] of patients. These symptoms significantly decrease the patients' quality of life [26]. Despite being a common occurrence in patients awareness of gastrointestinal disorders among doctors remains to be low [29].

\section{Diarrhea}

Dysfunctions of the small intestine, in case of diabetes complications, manifest primarily through chronic diarrhea, which may be accompanied by steatorrhea, caused by several mechanisms, e.g. a disorder of the exocrine function of the pancreas [30]. First report of diarrhea as a rare complication of diabetes was de- scribed by Joslin in 1912 [31]. Typical diabetic diarrhea has a chronic and intermittent nature $[8,28,30]$ with episodes of varied and unpredictable duration [30]. It is defined as a stool volume exceeding $200 \mathrm{ml}$ per day [28] with high frequency (up to 20 bowel movements in 24 hours), usually watery with a strong pressure on the stool [31]. In most patients diarrhea occurs in the evening at night or in the morning; occasionally is noctural $[9,30]$. Absence of pain is characteristic for this type of diarrhea [29, 31]. Its etiology comprises complex mechanisms described above which lead to, among others, motility disorders of the small intestine. In case of diarrhea, the microbiological factor and the small intestinal bacterial overgrowth (SIBO) $[8,21,27$, $28,30,32$ ] linked with it should always be taken into account. In research conducted by Bettin Z. et al. SIBO was diagnosed in over $1 / 3$ of patients with diabetes complicated by symptoms of autonomic neuropathy in respect to the circulatory system [32]. Diarrhea is considered to appear in patients with poorly controlled glycemia already accompanied by neuropathy $[9,28$, 29, 31]. Additionally, other mechanisms should be considered as etiology of it, such as exocrine pancreatic insufficiency, bile acid malabsorption [28, 29, 31], drugs being taken and their adverse effects, e.g. metformin $[29,33]$ or acarbose [33]. Consumption of diet products containing sorbitol or mannitol by the patient should also be excluded as a cause, as these artificial sweeteners can cause osmotic diarrhea as well $[28,33]$. In the course of diagnosis, gluten-sensitive enteropathy which occurs more frequently in patients with type 1 diabetes (up to $5 \%$ ), should also be excluded as a cause [33].

\section{Constipation}

Constipation is the most common symptom related to the gastrointestinal tract reported by patients with diabetes $[8,9,27-29,33]$, up to $60 \%[9,29]$. In a population study conducted by Maleki et al. [34] it was shown that $20-44 \%$ of patients with diabetes report symptoms of constipation or admit to taking laxatives more frequently. A severe form of constipation with megacolon occurs very rarely [29]; it may be accompanied by diarrhea (due to stool leakage) and fecal incontinence $[28,29]$. The exact etiology of constipation in patients with diabetes is not known [28]. One of the hypotheses assumes an influence of autonomic neuropathy and a lack of synchronization of mechanisms acting between intestinal muscles and anal sphincters [28]. However, the little research concerning the gastrointestinal transit time in patients with diabetes conducted shows contradictory results. For example, Hye-Kyung Jung et al. showed a longer gastrointestinal transit time in patients with diabetes 
compared to the control group; however, the research showed no correlation with symptoms of autonomic neuropathy in respect to the circulatory system [35], which in turn was demonstrated in two other pieces of research $[36,37]$. It should be remembered that proper defecation is a highly complex interaction between the anorectal motor and sensory functions: the rectum and the sigmoid colon need to be filled with proper fecal mass, proper distention of the rectum and the act of defecation, consisting of contraction of the abdominal press and relaxation of pelvic floor and rectal muscles at an appropriate time, must occur [38]. Any irregularities on various levels in this complex process result in constipation.

\section{Fecal incontinence}

Fecal incontinence is a very problematic and embarrassing symptom [29], which the patients do not report to a doctor for years. According to latest research, not only dysfunction of the anal sphincters, but also other factors such as: abnormal anorectal reflexes or rectal sensory-motor dysfunction [39] play a part in the process leading to fecal incontinence. Dysfunctions of both internal and external anal sphincters stemming from neuropathy may, during the course of diabetes, manifest themselves through fecal incontinence [27, 29]. In most patients with fecal incontinence and diabetes anorectal manometry shows reduced visceral sensitivity and lowered pressure in respect of the anal canal which is likely to be due to a dysfunction of the structures of the autonomic system in relation to the internal anal sphincter [28]. In the etiology of anorectal function disorders in diabetes leading to incontinence or constipation, a direct influence of elevated glucose concentration in blood is considered [29, 40,41]. In a research conducted by Russo et al. it was shown that high glucose concentration in blood in healthy volunteers affects functioning of anal sphincters and raises thresholds of visceral sensitivity [40]. In another research raised thresholds of visceral sensitivity in hyperglycemia were also demonstrated, which may significantly influence etiology of both fecal incontinence and constipation [41].

Over many years, a number of research concerning anorectal function in patients with diabetes were conducted, indicating disorders, most often in correlation with duration and occurrence of diabetic neuropathy $[10,12,42,43]$. A relation between anorectal function disorders and diabetic neuropathy has already been demonstrated in the 1950s [44]. For example, in a research conducted by Deen et al. a lack of or abnormalities in the rectoanal inhibitory reflex (RAIR) was demonstrated in all patients with fecal inconti- nence and diabetes. Abnormal visceral sensitivity in comparison with the control group was shown among these patients [43]. In other research irregularities were also found, e.g. in respect to the anal sphincters. In conclusion, it can be said that neuropathy, microangiopathy and myopathy developing during the course of complex mechanisms as complications of diabetes lead to anorectal function disorders in patients with diabetes [43]. These irregularities, though in a sense not directly life-threatening, do significantly lower the quality of life.

\section{Treatment}

Research conducted in recent years enables a different understanding of mechanisms leading to changes in the gastrointestinal tract in the course of diabetes. Until fairly recently autonomic neuropathy was thought to be the cause of these complications; today it is known that, apart from neuropathy, other pathophysiological factors, such as: abnormalities in the enteric nervous system, irregularities in ICC or myopathy of smooth muscles as well as abnormalities in the intestinal microflora [1, 4], also play an important role. Considering the above, and due to the complexity of the processes leading to diabetic gastroenteropathy, translating them into development of suitable methods of clinical treatment presents a considerable challenge [1]. For example, the significant role which oxidative stress plays in the etiology of gastrointestinal complications was discovered, but treatment attempts using antioxidants failed to bring satisfactory results [1].

Currently administered treatment is symptomatic. It is known that after the occurrence of the disorders related to DAN there is no treatment which would effectively eliminate their symptoms [7]. Recent research confirms that in case of type 1 diabetes mellitus, a proper insulin therapy slows down the development of DAN as well as peripheral neuropathy [45], whereas in type 2 diabetes certain antidiabetic drugs, e.g. GLP-1 agonists or thiazolidinediones demonstrate a positive effect in case of diabetic complications. There is also a research indicating negative effect of metformin on neuropathy through the vitamin B12 metabolism [7]. Treatment of each particular gastrointestinal symptom is difficult. In case of constipation proper hydration, diet and physical activity form the basis of treatment $[27,29]$. Various osmotic laxatives are also used $[9,28$, 29]. Dietary treatment should consist of the diet with soluble fibers (20-30 g daily). Insoluble fibers should be avoided, since, in case of concomitant gastroparesis, they may cause stomach ailments or bezoar formation. Certain patients may benefit from prokinetic drugs, e.g. cisapride [28]. Among new drugs, lubiprostone which 
works via type 2 chloride channels in enterocytes and stimulates water and electrolyte secretion thus influencing intestinal transit time $[9,29]$ is mentioned. Another drug - prucalopride, a selective $5 \mathrm{HT} 4$ receptor agonist [29], demonstrates a similar effect.

In case of diarrhea, treatment is mostly empirical and includes adequate fluid intake, supplementation of electrolytes and maintaining the best possible control of glycemia. Antidiarrheal drugs should be taken only on an ad hoc basis [27, 29].

It was demonstrated that patients with diabetes are often diagnosed with small intestinal bacterial overgrowth [32]. Rifaximin is the recommended drug in that case where it eradicates bacterial overgrowth in approximately $80 \%$ of patients $[9,29]$ and lessens the symptoms in 33-92\% of them [9]. In case of severe diarrhea resisting treatment, somatostatin analogs are used $[9,29]$. In treating diarrhea differential diagnosis and causal treatment are essential, e.g. in case of exocrine pancreatic insufficiency - supplementation of enzymes, etc. [9, 27, 28].

In fecal incontinence using diapers becomes necessary [28]. Antidiarrheal drugs may prove helpful loperamide, taken on an ad hoc basis [29].

\section{Summary}

The purpose of this article was to familiarize the reader with abnormalities concerning the lower gastrointestinal tract in patients with diabetes and to present the latest views related to their pathogenesis and possibilities of treatment as well as subjects of future research. According to the cited literature most patients with diabetes (up to 75\%) report gastrointestinal disorders, which is confirmed by the authors' clinical experience. We find that such symptoms as chronic constipation or diarrhea often negatively affect the patients' quality of life. The issue of fecal incontinence deserves particular attention, as it is an embarrassing problem and is often not reported by the patients, yet at the same time, in the authors' opinion, it occurs frequently, particularly in patients with prolonged, poorly controlled diabetes. Authors share the view expressed by Bajawa et al. concerning abnormalities in the anorectal function as a cause of these disorders. Based on clinical experience we find that female gender and a considerable obstetric history (multiple pregnancies, high birth weight of children and obstetric complications), as well as obesity, are significant additional risk factors for fecal incontinence in patients with diabetes. Russo et al. and Avasar et al. consider the issue of hyperglycemia and its influence on anorectal functions. During interviews, patients often report exacerbation of symptoms: fecal incontinence, diarrhea or constipation when diabetes is poorly controlled, which is consistent with research cited in the article. Therefore, considering the high complexity of processes underlying gastrointestinal disorders in diabetes, best possible diabetes control appears essential, as it may slow down the development of these disorders or reduce their severity. From a practical point of view reports of frequent occurrence of small intestinal bacterial overgrowth in patients with diabetes are significant. Gatopoulou et al. point out high treatment effectiveness of rifaximin, which is consistent with the authors' clinical experience. In the authors' opinion, empirical treatment of SIBO should be undertaken in every patient with diabetes and chronic diarrhea after other causes have been excluded. Unfortunately, there are no effective methods of treating diabetic gastrointestinal complications; treatment is still only symptomatic. However, discovery of new mechanisms in their pathogenesis gives hope that more effective methods of treatment will be developed in the future.

\section{Conflict of interest}

Authors do not report any conflict of interest.

\section{REFERENCES}

1. Yarandi SS, Srinivasan S. Diabetic gastrointestinal motility disorders and the role of enteric nervous system: current status and future directions. Neurogastroenterol Motil 2014; 26: 611-624.

2. Zalecenia kliniczne dotyczące postępowania u chorych na cukrzycę 2014. Diabetologia Kliniczna 2014; 3: A36.

3. Rossol S. Constipation in patients with diabetes mellitus. MMW Fortschr Med 2007; 149: 39-42.

4. Gregersen H, Liao D, Drewes AM et al. Ravages of Diabetes on gastrointestinal Sensory-motor Function: Implications for Pathophysiology and Treatment. Curr Gastroenterol Rep 2016; 18: 6.

5. Drzewoski J, Jarosińska-Wiśniewska M. Zaburzenia przewodu pokarmowego u chorych na cukrzycę. Pediatria Współczesna 2004; 6: 361-364.

6. Kempler P, Amarenco G, Freeman R. Management strategies for gastrointestinal, erectile, bladder, and sudomotor dysfunction in patients with diabetes. Diabetes Metab Res Rev 2011; 27: 665-677.

7. Verrotti A, Prezioso G, Scattoni R, Chiarelli F. Autonomic neuropathy in diabetes mellitus. Front Endocrinol 2014; 5: 205.

8. Vinik Al, Maser RE, Mitchell BD, Freeman R. Diabetic Autonomic neuropathy. Diabetes Care 2003; 26: 1553-1579.

9. Gatopoulou A, Papanas N, Maltezos E. Diabetic gastrointestinal autonomic neuropathy: Current status and new achievements for everyday clinical practice. Eur J Intern Med 2012; 23: 499-505.

10. Caviezel F, Bossi A, Baresi A, Salvini A. Anorectal manometry as evaluating test for impaired ano-rectal function in diabetes mellitus. Acta Diabetol Lat 1986; 23: 331-338.

11. Søfteland $E$, Brock C, Frøkjær JB et al. Rectal sensitivity in diabetes patient with symptoms of gastroparesis. J Diabetes Res 2014; 2014: 784841

12. Eldeskoy A, Bahgat M, El-Shreif M et al. Anorectal dysfunctions in diabetic autonomic neuropathy and microangiopathy. Arab Journal of Gastroenterology 2010; 11: 79-82.

13. Ordög T, Hayashi Y, Gibbons SJ. Cellular pathogenesis of diabetic gastroenteropathy. Minerva Gastroenterol Dietol 2009; 55: 315-343 
14. Chandrasekharan $B$, Srinivasan $S$. Diabetes and the enteric nervous system. Neurogastroenterol Motil 2007; 19: 951-960.

15. Sytze Van Dam P, Cotter MA, Bravenboer B, Cameron NE. Pathogenesis of diabetic neuropathy: Focus on neurovascular mechanisms. Eur J Pharmacol 2013; 719: 180-186.

16. Vazeou A, Papadopoulou A, Papadimitiou A et al. Autonomic neuropathy and gastrointestinal motility disorders in children and adolescents with type 1 diabetes mellitus. J Pediatr Gastroenterol Nutr 2004; 38: 61-65.

17. Wegener M, Börsch G, Schaffstein J et al. Gastrointestinal transit disorders in patients with insulin-treated diabetes mellitus. Dig Dis 1990; 8: 23-36.

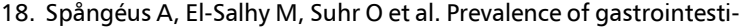
nal symptoms in young and middle-aged diabetic patients. Scand J Gastroenterol 1999; 34: 1196-1202.

19. Werth B, Meyer-Wyss B, Spinas GA et al. Non-invasive assessment of gastrointestinal motility disorders in diabetic patients with and without cardiovascular signs of autonomic neuropathy. Gut 1992; 33: 1199-1203.

20. de M Bandeira S, da Fonseca US, da S Guedes G et al. Oxidative Stress as an Underlying Contributor in the Development of Chronic Complications in Diabetes Mellitus. Int J Mol Sci 2013; 14: 3265-3284.

21. Takuma K, Fang F, Zhang W et al. RAGE-mediated signaling contribiutes to intraneuronal transport of amyloid-beta and neuronal dysfunction. Proc Natl Acad Sci USA 2009; 106: 20021-20026.

22. Kasznicki J, Kosmalski M, Sliwinska A et al. Evaluation of oxidative stress markers in pathogenesis of diabetic neuropathy. Mol Biol Rep 2012; 39: 8669-8678.

23. Al-Ani FS, Al-Nimer MS, Ali FS. Dyslipidemia as a contributory factor in etiopathogenesis of diabetic neuropathy. Indian Journal of Endocrinology and Metabolism 2011; 15: 110-114.

24. He CL, Soffer EE, Ferris CD et al. Loss of interstitial cells of Cajal and inhibitory innervation in insulin-dependent diabetes. Gastroenterology 2001; 121: 427-434.

25. Yoshiya K, Lapchak PH, Thai TH et al. Depletion of gut commensal bacteria attenuates intestinal ischemia/reperfusion injury. Am J Physiol Gastrointest Liver Physiol 2011; 301: G1020-1030.

26. Frokjer JB, Due Andersen S, Ejskjer $\mathrm{N}$ et al. Gut sensations in diabetic autonomic neuropathy. Pain 2007; 131: 320-329.

27. Shakil A, Church RJ, Rao SS. Gastrointestinal complications of diabetes. American Family Physician 2008; 77: 1697-1702.

28. Bekele G, Kabadi UM. Gastrointestinal manifestation of diabetes mellitus. Int J Diab Dev Countries 1996; 16: 54-58.

29. Krishnan B, Babu S, Walker J et al. Gastrointestinal complications of diabetes mellitus. World Journal of Diabetes 2013; 4: 51-63.
30. Bjelaković G, Nagorni A, Stamenković I. Diabetes mellitus and digestive disorders. Acta Fac Med Naiss 2005; 22: 43-50.

31. Joslin EP. Metabolism in diabetes mellitus. 1912 Washington DC: Carnegie Institution.

32. Zietz B, Lock G, Straub RH. Small-Bowel Bacterial Overgrowth in Diabetic Subjects Is Associated With Cardiovascular Autonomic Neuropathy. Diabetes Care 2000; 23: 1200-1201.

33. Sellin JH, Chang EB. Therapy Insight: gastrointestinal complications of diabetes - pathophysiology and management. Nat Clin Pract Gastroenterol Hepatol 2008; 5: 162-171.

34. Maleki D, Camilleri M, Zinsmeister AR, Rizza RA. Pilot study of pathophysiology of constipation among community diabetics. Dig Dis Sci 1998; 43: 2373-2378.

35. Hye-Kyung J, Doe-Young K, II-Hwan M, Young-Sun H. Colonic Transit time in diabetic patients - comparsion with healthy subjects and the effect of autonomic neuropathy. Yonsei Medical Journal 2003; 44: 265-272.

36. Werth B, Meyer-Wyss B, Spinas GA, Drewe J et al. Non-invasive assessment of gastrointestinal motility disorders in diabetic patients with or without cardiovascular signs of autonomic neuropathy. Gut 1992; 33: 1199-1203.

37. Kawagishi T, Nishizawa Y, Okuno Y, Sekiya K. Segmental gut transit in diabetes mellitus: effect of cisapride. Diabetes Res Clin Pract 1992; 17: 137-144.

38. Basilisco G, Coletta M. Chronic constipation: A critical review. Digestive and Liver Disease 2013; 45: 886-893.

39. Bajawa A, Emmanuel A. The physiology of continence and evacuation. Best Practice and Research Clinical Gastroenterology 2009; 23: 477-485.

40. Russo A, Sun WM, Sattawatthamrong $Y$ et al. Acute hyperglicaemia affects anorectal motor and sensory function in normal subjects. Gut 1997; 41: 494-499.

41. Avsar E, Ersoz O, Karisik E et al. Hyperglicemia-Induced attenuation of rectal perception depends upon pattern of rectal balloon inflation. Digestive Diseases and Sciences 1997; 42: 2206-2212.

42. Deen KI, Premaratna $\mathrm{R}$, Fonseka $\mathrm{M}$ et al. The recto-anal inhibitory reflex: Abnormal response in diabetic suggests an intrinsic neroenetropathy. Journal of Gastroenterology and Hepatology 1998; 13: 1107-1110.

43. Epanomertakis E, Koutsoumbi P, Tsiaoussis I et al. Impairment of Anorctal Function in Diabetes mellitus Pararellels Duration of Disease. Dis Colon Rectum 1999; 42: 1394-1400.

44. Martin M. Diabetic neuropathy: a clinical study of 150 cases. Brain 1958; 76: 594-624.

45. Lee SS, Han HS, Kim H. A 5-yr follow-up nerve conduction study for the detection of subclinical diabetic neuropathy in children with newly diagnosed insulin-dependent diabetes mellitus. Pediatr Diabetes 2010; 11: 521-528. 